\title{
Near infra-red and optical colour gradients in E-type galaxies
}

\section{Inferences on dust content ${ }^{\star, \star \star}$}

\begin{abstract}
R. Michard
Observatoire de Paris, LERMA, 77 Av. Denfert-Rochereau, 75015 Paris, France

e-mail: raymond.michard@obspm.fr

Received 6 September 2004 / Accepted 8 June 2005

Abstract. Colour gradients are considered for a sample of circa 50 E-type galaxies in the Local Supercluster. The new data includes isophotal colour profiles in $J-H, J-K, V-J$ and $V-K$, measured using 2MASS frames mostly from the Large Galaxies Atlas, $V$ frames from previous work and $V$ profiles from the literature. This is supplemented by $U-B, B-V, B-R$, $V-I$ colour gradients obtained anew from published photometric data. Colour gradients in E galaxies show remarkably large variations from object to object and do not correlate with other properties. Metallicity gradients are the primary cause as shown before. Age gradients with opposite effects are possibly needed to explain objects with small colour gradients. Some empirical evidence of such age effects has been found for a subset of objects with morphological peculiarities and younger stars mixed. Dust has only modest effects on colour gradients, as shown by the fact that objects with zero IRAS $100 \mu$ flux have the same average values of the gradients, except in $V-J$ and $V-K$, as those with non zero flux (cf. Table 7). This last subsample however exhibits poor but definite correlations between IRAS flux and gradients, which might be caused by the presence of a few relatively dusty galaxies in the sample. Given the absence of a correlation between any gradients and galaxy velocity dispersion (and hence mass), the observations do not agree with the predictions of the monolithic scenario for the formation of E galaxies. Simulated datasets of "dummy" objects mimicking the hierarchical scenario have been obtained, and used to test a technique for estimating the dust content of E-galaxies from the comparison of the $V-K$ (or $V-J$ ) colour gradients with the $U-B$ (or $B-V$ ) ones: the contents of diffuse dust, gauged in terms of published models, are obtained for a dozen objects.
\end{abstract}

Key words. galaxies: elliptical and lenticulars, CD - galaxies: ISM

\section{Introduction}

The colour profiles in E-type galaxies are an important source of information on radial stellar population variations, and perhaps also on the content of the diffuse dust and its distribution. From extensive UBR data by Franx et al. (1989, Fal89) and Peletier et al. (1990, Pa190) the latter authors favour an explanation of colour gradients as due to the decrease of the stellar metallicity outwards from the center of the object. On the other hand Wise \& Silva (1996) compare the same data to calculated colour gradients resulting from their theory of transfer in dusty models of galaxies, but without conclusive results. Goudfrooij et al. (1994a,b,c, Ga194) published an extensive survey of interstellar matter in a complete sample of ellipticals. Goudfrooij $\&$ de Jong (1995) have estimated from IRAS data the amount of dust in sample galaxies, and then used a model by

^ Based in part on observations collected at the Observatoire de Haute-Provence (OHP).

$\star \star$ Photometry data mentioned in Sect. 2 is only available in electronic form at the CDS via anonymous ftp to cdsarc.u-strasbg. fr (130.79.128.5) or via

http://cdsweb.u-strasbg.fr/cgi-bin/qcat?]/A+A/441/451
Witt et al. (1992, Wa192) to infer the $B-I$ colour gradients from this dust. In some cases these inferred gradients are of the same order as observed ones.

The radial profiles of line-indices directly prove the occurence of population gradients in ellipticals (Kobayashi \& Arimoto 1999), while these objects certainly contain some residual dust from their past episodes of star formation. Many authors have indeed "tracked" discrete dust features in ellipticals, (see references in Michard 1998). On the other hand, many such objects are undetected by IRAS at 60 and $100 \mu$ (Knapp et al. 1989). In order to ascertain the possible influence of diffuse dust on colour gradients, the above quoted data were re-discussed by Michard (2000, RM00): on average the relative values of gradients for various colours are fully compatible with calculated metallicity gradients but not with dust induced gradients.

It may be useful to reconsider this question, due to the availability of new significant data. Most important is the publication of the 2MASS images in the Extended Sources Catalogue and more recently in the Large Galaxies Atlas (Jarrett et al. 2003). From these well calibrated images we have derived $J-H$ and $J-K$ colour distributions, while the use of available $V$-light 
images or magnitude profiles allowed to calculate the $V-J$ and $V-K$ profiles. As regards the colours derived from the $U B V R I$ pass-bands, the observations by Idiart et al. (2002) (IMP02) represent an important addition. It was possible to gather $U-B$, $B-V, B-R, V-I, V-J, V-K, J-H$ and $J-K$ colour gradients for about 50 ellipticals, and to estimate the accuracy of the data, unfortunately far from homogeneous.

The Optical-Near IR colour profiles are essentially new information, except for the pionneering observations of Silva \& Elston (1994). We hope therefore that our material may cast some light on the interplay of various physical causes leading to the characteristic colour gradients in E-type galaxies. When successive generations of stars are forming in a galaxy, or protogalaxy, their prefered site of birth is likely to be the near-center region, where the ISM concentrates in the potential field. This leads to a metallicity gradient (larger Z stars near the center) and to an age gradient (younger stars near the center) with opposite effects on the colour gradients. Henry \& Worthey (1999) used line-strength indices to derive separate mean metallicity and mean age gradients in a sample of E-type objects and found an anti-correlation. It does not seem feasible to make a similar quantitative interpretation of broad-band colours, due to the so-called age-metallicity "degeneracy", and also because the eventual presence and distribution of residual dust is likely to affect colour gradients.

In this paper we evaluate the relative success of various hypotheses for the appearance of colour gradients in E objects, and for their present properties. The following questions are considered: are the colour gradients compatible with the "monolithic" scenario for the formation of ellipticals? Do they offer possible constraints on some aspects of the "hierarchical" build-up of these objects? Are the gradients more or less influenced by the presence of dust left over from their star formation and accretion?

Starting from the pioneering work of Larson $(1969,1974)$, various so-called "dynamical" and "chemo-dynamical" models have described the build-up of an E galaxy from an ad hoc seed gaseous object, with constant improvements in the physical and technical approachs. Metallicity gradients were calculated and colour gradients often used as tests of the models (Carlberg 1984a,b; Theis et al. 1992; Chiosi \& Carraro 2002; Kawata 2001a,b). In the most recent work, many properties of ellipticals are recovered, including metallicity gradients: these increase systematically with the mass, and are possibly too large at the upper mass range.

In the hierarchical scenario, E galaxies are formed by the merger of two spirals in a "major merger", and/or through several successive mergers with objects smaller than the main component (White \& Rees 1978; Barnes 1992; Barnes \& Hernquist 1996). A present day elliptical contains a majority of stars formed in a small group of primeval galaxies assembled in a common CDM halo and later merged. The morphology of the present day object may have greatly changed since the formation of its oldest stars. Recent efforts tend to follow the birth and destiny of CDM structures, followed by the production of galaxies within CDM haloes, and their evolution through merging and accretion of intergalactic material. This involves the simultaneous teatment of large domains in the universe, containing thousands of galaxies (Kauffmann et al. 1999, 2000; Hatton et al. 2003; Helly et al. 2003). Colour gradients in E galaxies are thus a quite complex problem, depending on the development of population gradients in primeval objects, modification of gradients through mergers, accretion effects, possible starbusts induced by the mergers: the individual history of interactions for each object determines the present day properties. Large scale simulations of the hierarchical scenario reaching sufficient resolution to provide the distributions of populations within galaxies are not yet many (Steinmetz \& Navarro 2002; Kobayashi 2004). The latter author has published a "GRAPE-SPH chemo-dynamical simulation of elliptical galaxies" providing metallicity gradients for 128 objects including $72 \mathrm{E}$ galaxies and 48 dwarfs. It reproduces well the great variety of observed population gradients.

It is known that some dust is present in ellipticals, although in small relative amounts as compared with other Hubble types, and may influence colour gradients, strongly in such bands as $V-J$ or $V-K$, but also in others. In Sect. 4 we present simulations designed to estimate the mean amount of diffuse dust in the sample objects. The criteria are comparisons between dustfree and dust affected mock galaxies, with populations mimicking those expected from the hierarchical scenario.

\section{Often used notations}

- $r$ isophotal radius. $r=(a b)^{1 / 2}$ for an ellipse of semi-axis $a$ and $b . r_{\mathrm{e}}$ effective radius, i.e. of the isophote enclosing half the total light.

- $\Delta_{U B}$ colour gradient in $U-B . \Delta_{U B}=\mathrm{d}(U-B) / \mathrm{d}(\log r)$ and similar for other colours.

- $d i \mathrm{E}$, bo $\mathrm{E}$, unE subclassification of E galaxies as disky, boxy and undetermined.

- SSP Simple Stellar Population of unique age and metallicity.

- $\tau_{V}$ or Tau( $\left.V\right) V$ central optical thickness in Witt et al. (1992) models (Wa192).

\section{Collecting the gradients}

\subsection{Derivation of Visual-Near IR colour gradients}

\subsubsection{Techniques}

Our preferred technique is to measure directly isophotal colours on a pair of calibrated frames in the two involved passbands. For the colours $J-H$ and $J-K$ the 2MASS catalogues provide carefully calibrated frames, either in the Large Galaxies Atlas (Jarrett et al. 2003) or the Extended Sources Catalogue. For $V-J$ and $V-K$ we use $V$-band images from the Observatoire de Haute-Provence (Michard \& Marchal 1994; Idiart et al. 2002; HYPERLEDA data base). The set of isophotes to be used as template is derived following the classical technique (Carter 1978), involving ellipse-fitting and harmonic analysis of the deviations from the ellipse. It is applied either to the $V$ frame or to an average near IR frame obtained by summing the 3 images from 2MASS.

For objects without an available $V$ frame, we use instead a $V$-profile $\mu_{V}(r)$ taken from Goudfrooij et al. (1994, Gal94): these have been revised as described by RM00, eventually 
modifying the calibration and the adopted sky level, if judged appropriate. The $V-J$ and $V-K$ colour profiles are then derived from the comparison of the adopted $V$-profile to the $J$ and $K$-profiles measured upon 2MASS frames.

Whether images or $V$-profiles are used, the derivation of the colour profiles and gradients is a partly interactive operation: the observer is able:

- to adjust the adopted sky background level (in one or both the pass-bands), in order to cancel out the well known effects of imperfect sky substraction: this induces in the outer range of Colour-log $r$ profiles abrupt departures of the overall linearity which prevails at average $r$ values. Such ad hoc adjustments, which should obviously remain smaller than probable errors in the initial sky background, have been made and briefly discussed before (RM00, IMP02). Examples are given in Fig. 1;

- to select the inner limit of $r$ for the fit of the representative straight line which gives the gradient. One should avoid the effect of "differential seeing" (Michard 1999 or RM99) resulting from the different PSFs in the two frames used for a colour estimate. This effect has been taken care of, either by introducing an inner cut off in the profiles (Fal89; Pa190; Ga194), or by equalizing the PSFs through convolving or deconvolving one of the frame (RM99; IMP02). The 2MASS frames have generally nearly equal PSFs, but their sampling is coarser and their resolution worse than for the comparison $V$ data (except for part of the OHP frames). The Gal94 $V$-profiles are often of "too good" resolution compared to the 2MASS frames, so that the range of $r$ where the $V-J, K$ colours are badly affected is large, and one has to apply a rather severe inner cut-off. We also tried to avoid the range of $r$ affected by significant dust patterns, (Goodfrooij et al. 1994b; RM99), enlarging the inner cutoff for this purpose;

- to select the outer limit of $r$ where noise or residual background uncertainties preclude use of the data in the fit.

In the many instances of repeated fits with different $V$ data, these were averaged and the zero-point was evaluated for a standard radius of $r_{\mathrm{e}} / 2$. The colours were corrected using for the galactic extinction the $A_{\mathrm{g}}$ values from the RC3 with the coefficients by Rieke \& Lebofsky (1985), and the K-corrections by Poggianti (1997).

The sample of Visible-Near IR colours and gradients so derived contains 54 galaxies in the Local Supercluster, essentially selected on the basis of apparent size. All are E-type, except NGC 3115 and 5866. Only $J-H$ and $J-K$ were measured for NGC 3585. These data are to be included with the comparison UBVRI colours in Table A1, to be made available at the CDS Data Center.

\subsection{Derivation of UBVRI colour gradients}

It was tried to obtain a nearly complete set of $U-B, B-V$, $B-R$ and $V-I$ colours for objects of the above sample by merging the data from the above quoted surveys (Fal89; Pal90; Ga194; and IMP02). The first two provide the $U B R$ photometry, Gal94 the $B V I$ profiles and only IMP02 is nearly complete in the 5 pass-bands. This obviously precludes the assembly of a homogeneous dataset.

The colour gradients and intercept values were not taken directly from the published papers, but derived anew from the authors' photometry available at the CDS Data Center. The calibration were checked and the sky background levels reconsidered as in RM00. Also the cut-off inner and outer radii in the calculation of gradients were selected anew.

Among the objects of the above Visible-Near IR sample, the compilation provided $41 U-B$ colours, $53 B-V, 43$ $B-R$ and $47 V-I$. The weight of each compiled value varies between 1 and 3 depending on the number of independent sources. The excellent correlation (see below) between the $\Delta_{B V}$ and $\Delta_{B R}$ values for the IMP02 sample, has been assimilated to a physical relation: it was therefore used to supplement the $\Delta_{B R}$ list from available $\Delta_{B V}$ values and conversely. The $\Delta_{B V}$ and $\Delta_{B R}$ figures in the compilation are not fully independent.

\subsection{Errors}

\subsubsection{Ways to error estimates}

Formal errors sometimes obtained in the least-square fits are not of much value because the main sources of errors in deriving colour gradients affect large portions of the radial range. i) There are small residual errors in the evaluation of the sky background level (which is never completely flat). ii) There are distorsions in the colour profile due to the differences in the PSFs of the two frames used. As a result the errors in colour gradients measurements are generally quite large. The following tests are feasible:

- measure the studied quantity with two independent sources. For instance, between the IMP02 data and those derived from Pal90 there are about 20 galaxies in common: this allows a direct comparison between the same gradients $\Delta_{U B}$, $\Delta_{B R}$ obtained from the two sources. They can be crosscorrelated to check for the existence of systematic errors, to be eventually corrected if deemed significant. Similarly there are many objects in common between the Gal94 source and the IMP02 one (22 in $\Delta_{B V}$, and 15 in $\Delta_{V I}$ );

- correlate two colour-gradients, such as $\Delta_{U B}$ and $\Delta_{B V}$ in a given dataset. These are largely jeopardized by errors of measurements, but perhaps also by different responses of the two indices to fluctuations in astrophysical variables, say mean stellar metallicity or dust content. The study of "inside correlations", for instance $\Delta_{U B}$ against $\Delta_{B V}$, allows one to evaluate the probable error in $\Delta_{U B}$ if the other one is known, and if the possible cosmic scatter in the correlation is negligible;

- compare the dispersions of a given correlation in two datasets: their ratio gives an approximation of the ratio of the respective errors in these two datasets. Thus the errors in the $\Delta_{U B}, \Delta_{B R}$ from Pal90 can be found in terms of the errors of the same gradients in IMP02. 

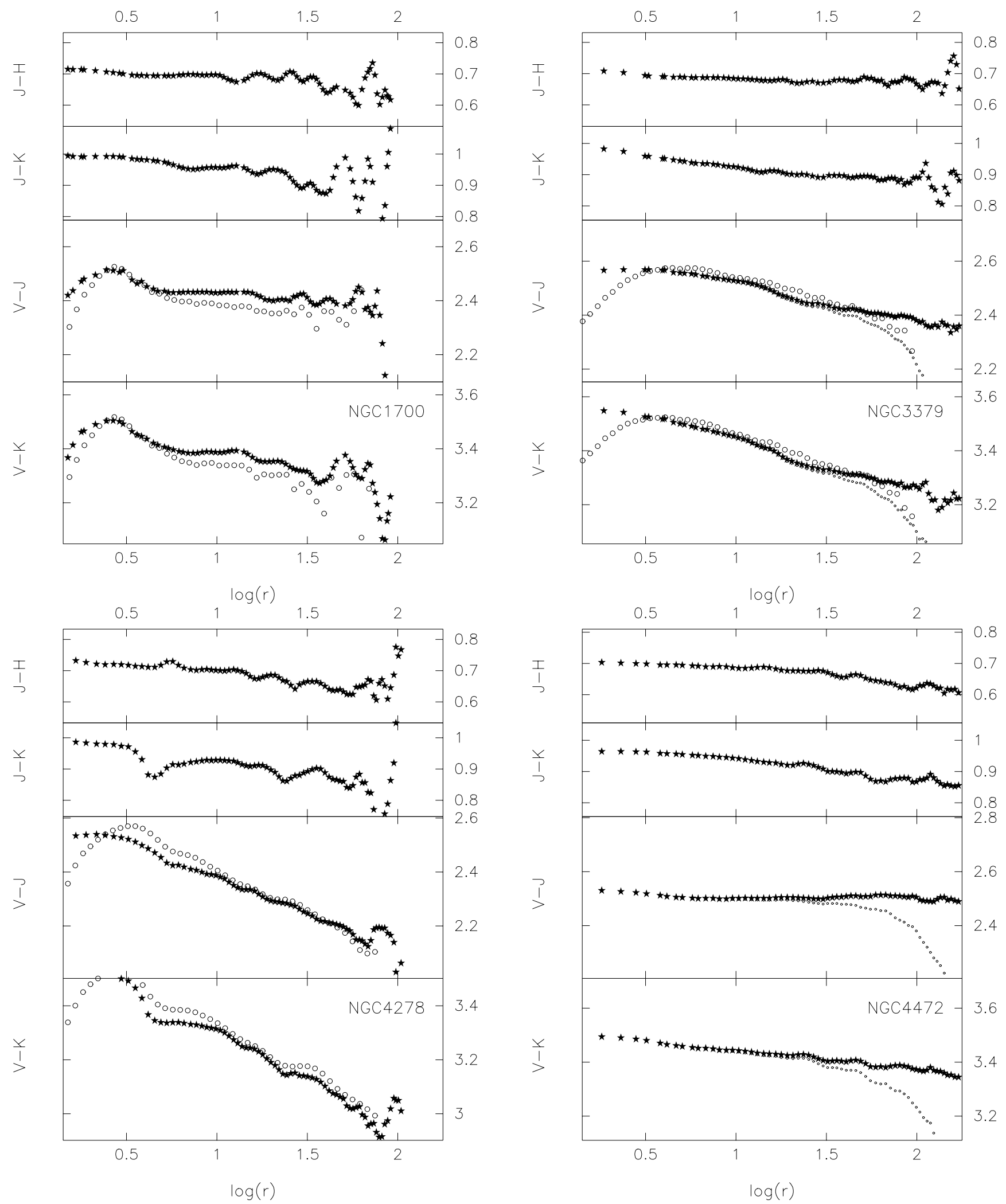

Fig. 1. Examples of the radial distribution of Visual-Near IR colours, derived from 2MASS frames and published $V$-images or profiles. Abscissae: $\log r$. Ordinates from top to bottom, $J-H, J-K, V-J$ and $V-K$ colours. $V$ data are from OHP frames, either before background adjustment (dots) or after (stars), and from Gal94 profiles (circles). In NGC 1700 and 4278 note the large effects of "differential seeing" on $V-J$ and $V-K$ gradients, specially for the Gal94 profiles derived from sharper images. For the very large objects NGC 3379 and 4472 , background corrections are needed to compensate small truncatures of the galaxy light in the 2MASS mosaics: these corrections are small, i.e. $0.4 \%$ in $J$ and $0.15 \%$ in $K$ in the difficult case of NGC 4472 . 


\subsubsection{Derivation of errors estimates in Visual-Near IR colours}

No specific study of the errors in the zero point colours $V-J$, $V-K, J-H$ and $J-K$ has been made because they are dominated by calibration errors, as are the integrated colours within $A_{\mathrm{e}}$, measured for a larger sample by Michard (2005). Probable errors of 0.025 in $V-J, V-K$ and 0.013 in $J-H$, $J-K$ were found there.

The following information has been used to derive errors in the Visual-Near IR colour gradients:

- for a number of objects, the $V-J, V-K$ colours and gradients can be obtained using both the OHP frames and the Gal94 profiles, providing two largely independent estimates. Calling $x_{1}=\Delta_{V J}$ with $V-J$ from the first source and similarly $x_{2}$ with $V-J$ from the second one, we find for 13 objects a mean $x_{1}-x_{2}$ of $0.015 \pm .013$ with $\sigma=0.045$. Assuming the errors to be the same for both sources, the probable error for $x_{1}$ or $x_{2}$ is 0.032 . The same exercice for $\Delta_{V K}$ gives an error of 0.037 . Similar results have been obtained in statistics of the $V-V$ pseudo-colour, where one $V$-magnitude profile is taken fron $\mathrm{Ga} 194$, the other from IMP02;

- the gradients $\Delta_{V J}$ and $\Delta_{V K}$ are very well correlated (slope 1.00 , correlation coefficient $\rho=0.975$ ) and it can be safely assumed that the residual $\sigma=0.034$ of this correlation is due only to errors of measurements. Because these are partly correlated in the two colours, we estimate the common probable error in the two gradients to reach the same value of $\sigma=0.034$, in excellent agreement with the above result;

- Again the gradients $\Delta_{J H}$ and $\Delta_{J K}$ are well correlated, with $\rho=0.47$ and $\sigma=0.019$. The same line of reasoning as above leads to a probable error of 0.017 for these two gradients.

\subsubsection{Derivation of error estimates in UBVRI colours}

The errors in the zero point colours $U-B, B-V, B-R, V-I$ mostly result from the available calibrations and may vary from object to object. Errors for the northern subsample are given in IMP02. The list will be completed in our full tabulated results available at the CDS Data Center (Table A1).

The following results have been obtained, along the above lines, for the estimation of the errors in UBVRI colour gradients:

- The correlation between gradients in IMP02 and the same from other sources are rather poor. Linear fits were calculated, but it was not deemed reasonable to use such loose correlations in merging the data. Residuals from the $45^{\circ}$ line are given in Table 1 . The IMP02 $\Delta_{V I}$ may be significantly underestimated as compared to Gal94, due to the "red halo" in the used CCD, a defect laboriously measured (Michard 2002) and corrected for. The $\Delta_{U B}$ from Pal90 may be also larger than in IMP02. These possible systematic effects were however neglected in the final compilation. Assuming the probable errors for IMP02
Table 1. Residuals between datasets from different sources. These are given as $X_{2}-X_{1}$ where $X$ is the studied variable from the data sources 1 and 2.

\begin{tabular}{llllll}
\hline \hline $\mathrm{X}$ & $\mathrm{S} 1$ & $\mathrm{~S} 2$ & $N$ & $m_{X}$ & $\sigma$ \\
\hline$\Delta_{V V}$ & $\mu_{V}(1)$ & $\mu_{V}(2)$ & 13 & -0.014 & 0.044 \\
$\Delta_{V J}$ & $\mathrm{~d} V J(1)$ & $d V J(2)$ & 14 & 0.015 & 0.045 \\
$\Delta_{V K}$ & $\mathrm{~d} V K(1)$ & $d V K(2)$ & 14 & 0.015 & 0.047 \\
$\Delta_{B V}$ & $\mathrm{IMP02+}$ & $\mathrm{Ga} 94$ & 21 & -0.003 & 0.028 \\
$\Delta_{V I}$ & $\mathrm{IMP02+}$ & $\mathrm{Ga} 94$ & 15 & -0.019 & 0.034 \\
$\Delta_{B R}$ & $\mathrm{IMP02+}$ & $\mathrm{Pa} 90$ & 18 & -0.010 & 0.030 \\
$\Delta_{U R}$ & $\mathrm{IMP02+}$ & $\mathrm{Pa} 90$ & 18 & -0.010 & 0.065 \\
\hline
\end{tabular}

(1) Variable $X$; (2), (3) sources S1, S2 with abbreviated keys: $\mu_{V}(1)$, $V$-profile from OHP frames; $\mu_{V}(2), V$-profile from Gal94; $\mathrm{d} V J(1)$, $\Delta_{V J}$ from 2MASS and OHP frames; $\mathrm{d} V J(2), \Delta_{V J}$ from 2MASS and Gal94 $V$-profiles; $\mathrm{d} V K(1)$ and $\mathrm{d} V K(2)$, same sources for $\Delta_{V K}$. Source IMP02 contains data from this reference and from miscellaneous OHP frames; (4) $N$ number of data; (5) $m_{X}$ mean residual; (6) standard deviation $\sigma$.

Table 2. "Inside correlations" between gradients from various sources, used in the estimation of probable errors in each dataset. The impartial correlation $Y=p X+a$ is given.

\begin{tabular}{llllcrll}
\hline \hline $\mathrm{X}$ & $\mathrm{Y}$ & $\mathrm{S}$ & $N$ & $p$ & $a$ & $\rho$ & $\sigma$ \\
\hline$\Delta_{B V}$ & $\Delta_{U B}$ & $\mathrm{I}$ & 39 & $2.10 \pm .23$ & $-.005 \pm .005$ & .75 & .029 \\
$\Delta_{B V}$ & $\Delta_{V I}$ & $\mathrm{I}$ & 39 & $1.12 \pm .16$ & $.022 \pm .003$ & .51 & .020 \\
$\Delta_{B V}$ & $\Delta_{B R}$ & $\mathrm{I}$ & 37 & $1.33 \pm .10$ & $.004 \pm .002$ & .90 & .012 \\
$\Delta_{B V}$ & $\Delta_{V I}$ & $\mathrm{G}$ & 32 & $1.39 \pm .30$ & $.030 \pm .006$ & .45 & .035 \\
$\Delta_{B R}$ & $\Delta_{U R}$ & $\mathrm{P}$ & 25 & $2.00 \pm .29$ & $.083 \pm .010$ & .73 & .051 \\
\hline
\end{tabular}

(1) $X$ variable; (2) $Y$ variable; (3) $\mathrm{S}$ source reference abbreviated as I for IMP02 (enlarged from several OHP measurements), G for Gal94, P for Pal90 and Fal89 merged; (4) number of objects $N$ after rejection of outliers; (5) slope $p$; (6) zero point $a$; (7) coefficient of correlation $\rho$; (8) dispersion $\sigma$.

gradients, estimates for the other subsets are readily obtained.

- The "inside correlations" for various data subsets are given in Table 2. The dispersion of data around the calculated regressions are clearly better than the dispersions around the $45^{\circ}$ line found in the comparison of different datasets and shown in Table 1. It appears therefore that errors in the derivation of gradients for various colours in a given experiment are correlated.

The "inside correlations" are relatively good for the IMP02 data: starting from a value of 0.01 for the error on $\Delta_{B V}$ (or $\Delta_{B R}$ ) the other errors are readily derived as .017 in $\Delta_{V I}$, 0.022 in $\Delta_{U B}, 0.032$ in $\Delta_{U R}$. Then the comparisons of residuals for various "inside correlations" allow to estimate the errors in other data subsets. These estimates are collected in Table 3, and are probably lower limits: if one tries to predict the dispersions in dataset comparisons (Table 1) from the estimated "inside" errors (Table 3 ), the predictions are systematically too low.

The errors for the weighted averages vary from object to object and will be given in Table A1, electronically available at CDS. Approximate mean errors, sufficient for the discussion below, are 0.027 in $\Delta_{U B}, 0.013$ in $\Delta_{B V}, 0.015$ in $\Delta_{B R}, 0.021$ in $\Delta_{V I}$. 
Table 3. Estimated mean errors in UBVRI gradients for various datasets.

\begin{tabular}{lll}
\hline \hline $\mathrm{X}$ & Source & $\sigma$ \\
\hline$\Delta_{B V}$ & IMP02+ & 0.010 \\
$\Delta_{B V}$ & Ga194 & 0.017 \\
$\Delta_{V I}$ & IMP02+ & 0.017 \\
$\Delta_{V I}$ & Gal94 & 0.025 \\
$\Delta_{B R}$ & IMP02+ & 0.012 \\
$\Delta_{B R}$ & PF++ & 0.020 \\
$\Delta_{U B}$ & IMP02+ & 0.022 \\
$\Delta_{U B}$ & PF++ & 0.035 \\
$\Delta_{U R}$ & IMP02+ & 0.030 \\
$\Delta_{U R}$ & PF++ & 0.040 \\
\hline
\end{tabular}

(1) Gradient studied $X$; (2) source of data; (3) estimated mean error $\sigma$. The source $\mathrm{PF}++$ results from merging the Fal89 and Pal90 data; IMP02+ contains a few OHP images besides those treated in the refered paper.

\section{Properties of colour gradients}

\subsection{Statistics of colour gradients}

\subsubsection{Distributions of colour gradients}

In Table 4 are presented the usual descriptive parameters of the distribution of gradients in the 8 studied colours. We have added the probable errors of measurement estimated above, distinctly smaller than the standard deviations. These distribution are shown in the histograms of Fig. 2. Some experiments have been made to test the significance of the calculated parameters: rejection of the very dusty S0 NGC 5866; division of the sample in two subsamples; consideration of the sample IMP02+ alone (optical colours only); first order correction of $\Delta_{V I}$ for the possible systematic error in the IMP02+ source. It appears that the Mean and Standard deviation are reliable (with a possible error of 0.008 for the Average of $\Delta_{V I}$ ). The skewnesses of the $\Delta_{V J}$ and $\Delta_{V K}$ distributions are rather robust, but the one for the $\Delta_{V I}$ is not. The distributions of the $\Delta_{U B}, \Delta_{B V}$, $\Delta_{B R}, \Delta_{J H}$ and $\Delta_{J K}$ do not differ much from Gaussian, although it is perhaps significant that the skewness parameter remains negative for all colours.

The distributions of the gradients $\Delta_{U B}, \Delta_{B V}, \Delta_{B R}, \Delta_{V K}$ and $\Delta_{J K}$ are clearly truncated at values above zero (neglecting the minus sign). This is not the case for $\Delta_{V I}$ and $\Delta_{V J}$. In the first case, errors of measurement are probably responsible: only one object, i.e. NGC 3250 gives a value at more than $3 \sigma_{\text {err }}$ from the mean. For $\Delta_{V J} 4$ objects are at more than 2.9 and 2 at more than $3 \sigma_{\text {err }}$ : this is of course not excluded by statistics, but it is striking to find so small a $\Delta_{V J}$ in NGC 4472 (cf. Fig. 1) and 3 others. The results for NGC 4472 have been checked and confirmed using other photometric data.

Pal90 showed that the ratios of $U-R$ to $B-R$ colour gradients can be explained through a metallicity gradient. The argument was reinforced in RM00 by considering a set of 4 colours. In the present work are collected 8 colours for nearly 50 objects. What becomes of the metallicity argument? As before we compare the observed relative values of the gradients with predicted values for metallicity variations according to the
Table 4. Parameters of the colour gradients distributions.

\begin{tabular}{llllllll}
\hline \hline Grad. & $N$ & Min & Max & Mean & $\sigma$ & Skew & $\sigma_{\text {err }}$ \\
\hline$\Delta_{U B}$ & 41 & -0.08 & -0.25 & -0.157 & .042 & $-0.52 \pm .6$ & .027 \\
$\Delta_{B V}$ & 53 & -0.02 & -0.12 & -0.066 & .022 & $-0.32 \pm .5$ & .013 \\
$\Delta_{B R}$ & 43 & -0.03 & -0.13 & -0.082 & .028 & $-0.27 \pm .6$ & .015 \\
$\Delta_{V I}$ & 47 & -0.03 & -0.16 & -0.061 & .036 & $-1.12 \pm .6$ & .021 \\
$\Delta_{V J}$ & 53 & -0.01 & -0.35 & -0.136 & .092 & $-1.15 \pm .5$ & .032 \\
$\Delta_{V K}$ & 53 & -0.07 & -0.52 & -0.227 & .112 & $-1.48 \pm .5$ & .035 \\
$\Delta_{J H}$ & 54 & -0.01 & -0.11 & -0.054 & .026 & $-0.51 \pm .5$ & .017 \\
$\Delta_{J K}$ & 54 & -0.04 & -0.17 & -0.093 & .033 & $-0.51 \pm .5$ & .017 \\
\hline
\end{tabular}

(1) Gradient $\Delta_{A B}$ of colour $A-B$. (2) Number $N$ of objects. (3) Minimum (one value in $V-I$ rejected). (4) Maximum. Both are averages of two extreme values (NGC 5866 rejected). (5) Mean. (6) Standard deviation $\sigma$. (7) Skewness and its probable error from the formula in Numerical Recipes (Press et al. 1992). (8) Probable errors of measured gradients $\sigma_{\text {err }}$. No rejections are made for the calculation of the parameters in Cols. (5)-(7).

Table 5. "Inside correlations" between gradients from new and merged data. The relation $Y=p X+a$ is given (taking errors in account).

\begin{tabular}{llllrlll}
\hline \hline$X$ & $Y$ & $N$ & $p$ & \multicolumn{1}{c}{$a$} & $\rho$ & $\sigma$ & \multicolumn{1}{l}{$\sigma_{0}$} \\
\hline$\Delta_{B V}$ & $\Delta_{U B}$ & 39 & $1.81 \pm .25$ & $-.038 \pm .005$ & .56 & .032 & .034 \\
$\Delta_{B V}$ & $\Delta_{V I}$ & 43 & $1.32 \pm .16$ & $.031 \pm .003$ & .63 & .023 & .025 \\
$\Delta_{B V}$ & $\Delta_{B R}$ & 39 & $1.33 \pm .05$ & $.002 \pm .001$ & .97 & .006 & .020 \\
$\Delta_{B V}$ & $\Delta_{V J}$ & 51 & $3.49 \pm .44$ & $.104 \pm .009$ & .48 & .067 & .039 \\
$\Delta_{B V}$ & $\Delta_{V K}$ & 51 & $3.95 \pm .48$ & $.047 \pm .010$ & .53 & .074 & .044 \\
$\Delta_{B V}$ & $\Delta_{J K}$ & 51 & $1.28 \pm .18$ & $-.004 \pm .004$ & .28 & .027 & .021 \\
\hline
\end{tabular}

(1) $X$ variable; (2) $Y$ variable; (3) number of objects $N$ after rejection of outliers; (4) slope $p$; (5) zero point $a$; (6) coefficient of correlation $\rho$; (7) dispersion $\sigma$; (8) expected dispersion $\sigma_{0}$ from estimated errors.

calculations of SSP models from three authors (Worthey 1994; Bressan et al. 1994; Bruzual \& Charlot 2003). The pure effect of diffuse dust is also considered for models of dusty ellipticals from Wal92. The results are given in Table 6. The relative values of $\Delta_{U B}$ and $\Delta_{B V}$ are well recovered by the various population models. Such ratios at longer wave-lengths disagree between themselves and often also with the observations. Gradients induced by diffuse dust have the wrong gradients ratios in all colours. It appears that, on average, metallicity gradients remain the best supported explanation of the colour gradients in E galaxies, although contributions of dust remain possible.

\subsubsection{Correlations between gradients in various colours}

A series of correlation diagrams between $\Delta_{B V}$ and the gradients in other pass-bands is shown in Fig. 3, and the usual parameters describing the correlations are collected in Table 5. It appears that all gradients are correlated, but not very tightly, with the one taken as reference. Although the data come from a compilation of heterogeneous material (see above for its discussion), the $\Delta_{U B}$ and $\Delta_{V I}$ are as well correlated with $\Delta_{B V}$ than shown by IMP02. The excellent correlation of $\Delta_{B R}$ with the reference has been partly "built in" the data by our technique for 

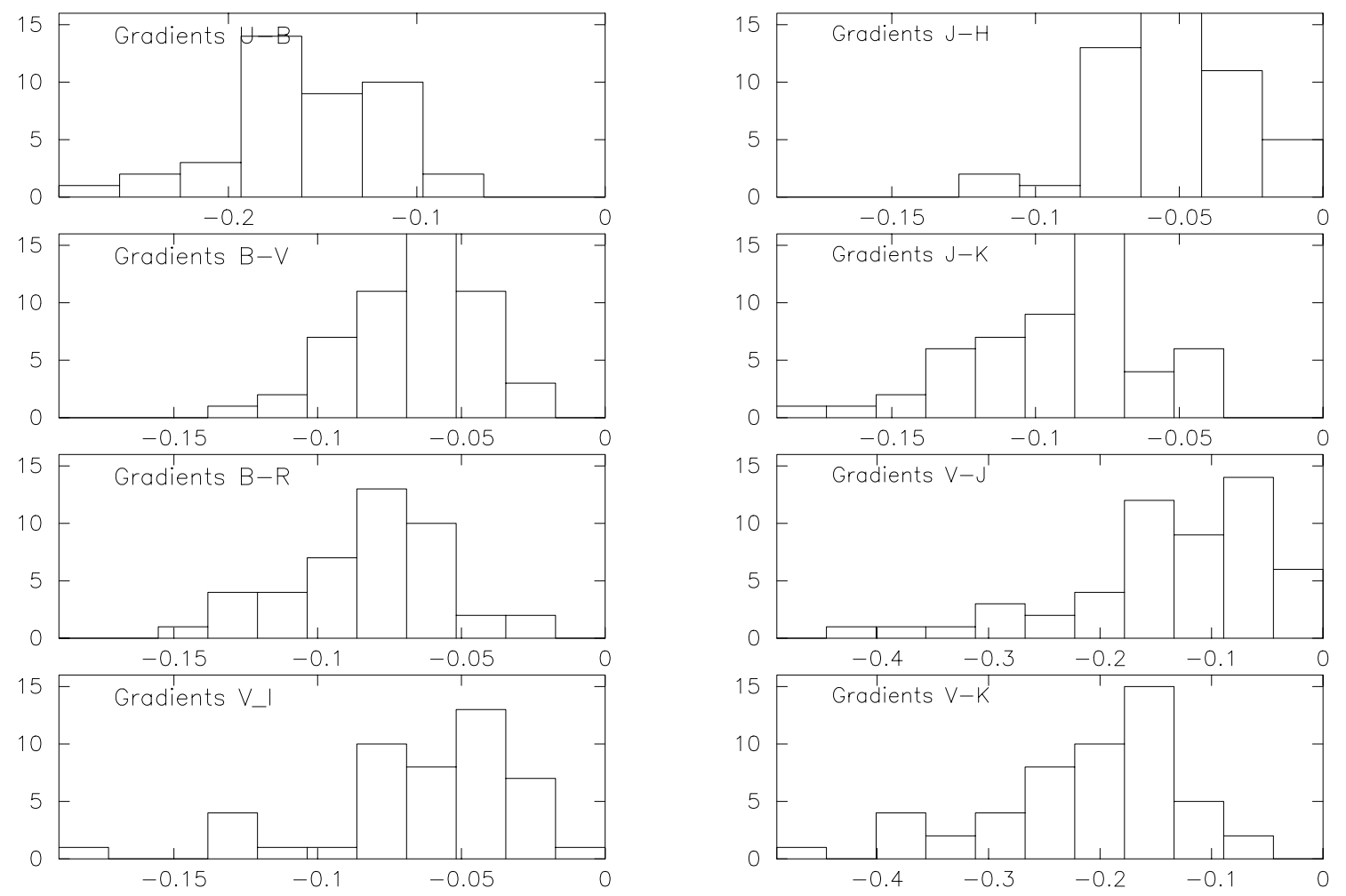

Fig. 2. Histograms of the distributions of the gradients of "optical" colours.

Table 6. Relative gradients, observed and calculated: $\Delta_{B V}$ is taken as unit.

\begin{tabular}{llllllll}
\hline \hline Source & $U-B$ & $B-V$ & $B-R$ & $V-I$ & $V-J$ & $V-K$ & $J-K$ \\
Obs. & 2.38 & 1.00 & 1.24 & 0.92 & 2.06 & 3.44 & 1.41 \\
\hline W94 & 2.45 & 1.00 & 1.48 & 1.17 & 4.24 & 5.14 & 0.90 \\
Ba194 & 2.57 & 1.00 & 1.86 & 1.68 & 5.36 & 6.68 & 1.32 \\
BC04 & 2.03 & 1.00 & 1.58 & 1.18 & 3.36 & 3.76 & 1.18 \\
\hline Wal92 & 0.32 & 1.00 & 2.17 & 3.17 & 5.25 & 8.11 & 2.86 \\
id & 0.92 & 1.00 & 2.45 & 3.83 & 6.31 & 9.75 & 3.44 \\
\hline
\end{tabular}

Sources: Obs. Mean observed gradients from Table 4; W94 calculated from Worthey (1994), range $[\mathrm{Fe} / \mathrm{H}]-0.25$ to 0.25 , age $12 \mathrm{Gyr}$; Bal94 calculated from Bressan et al. (1994), range $Z=0.008$ to 0.05, age 12.56 Gyr; BC03 calculated from Bruzual \& Charlot (2003) $Z=0.008$ to 0.05 , age $12 \mathrm{Gyr}$; Wal92 dust induced colour gradients calculated from Witt et al. (1992), Table 3E, $\operatorname{Tau}(V)=4$ (1st. line) and $\operatorname{Tau}(V)=1$ (2nd. line). The following columns show the relative gradients $\Delta_{U B} / \Delta_{U V}$ for $U-B, B-V, \ldots$.

merging the $B-R$ results of Fal89 or Pal90 with the $B-V$ from Gal94 (see above).

The dispersions about the impartial regression lines are similar to the dispersions predicted from the adopted mean errors in the data. They are somewhat larger however for $\Delta_{V J}$, $\Delta_{V K}$ or $\Delta_{J K}$. There are several possible reasons to this fact: i) besides the dominant effect of the metallicity gradients, age gradients are also present, and various colours do not "respond" in the same way to these parameters: the "degeneracy" noted in W94 is not complete; ii) for some colours, notably $V-J$ and
$V-K$, the effects of dust may affect the metallicity-induced correlation. These effects will be discussed quantitatively in Sect. 4.

\subsection{Colour gradients and other galaxy properties}

\subsubsection{Searching for correlations between galaxy mass and gradients}

Since monolithic theories of E-galaxy formation (Chiosi \& Carraro 2002) predict an increase of the metallicity gradient $\mathrm{dZ} / \mathrm{d}(\log r)$ with galaxy mass, it seems useful to check for an hitherto undetected correlation between the colour gradients and the central velocity dispersion $\log \sigma_{0}$ (taken from Michard \& Prugniel 2004): these observables are mostly dependent from the two quoted physical parameters. Figure 4 gives an example of the correlation diagram of a colour gradient, i.e. $\Delta_{U V}$, with $\log \sigma_{0}$; the diagrams for other colours are of similar appearance. There is no clear correlation, but, if anything, a trend for the objects with the larger $\log \sigma_{0}$, i.e. the most massive, to have the smaller colour gradients. This result contradicts the predictions of monolithic models.

Kobayashi \& Arimoto (1999) studied correlations between the Lick line-indices gradients and the mass, as measured by the central velocity dispersion, and found none. Unfortunately no significant correlation appears between their $\mathrm{Mg}_{2}$ or $\mathrm{Mg}_{b}$ gradients and the present colour gradients, a rather disappointing result, most probably due to observational errors. 

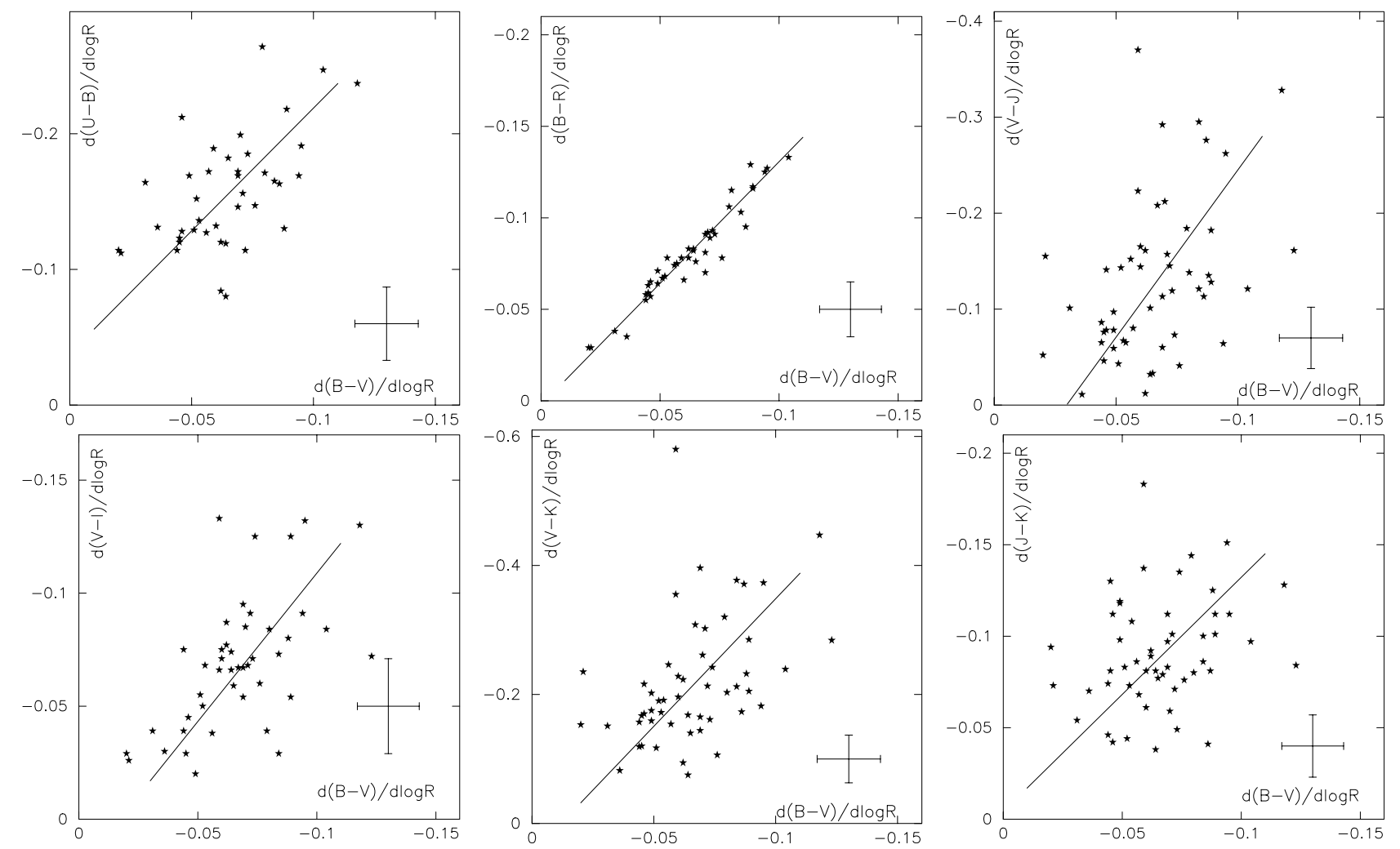

Fig. 3. Correlation diagrams between $\Delta_{B V}$ and gradients in other colours, with calculated regressions (Table 5) and indications of the estimated average errors (Table 4). See comments in text.

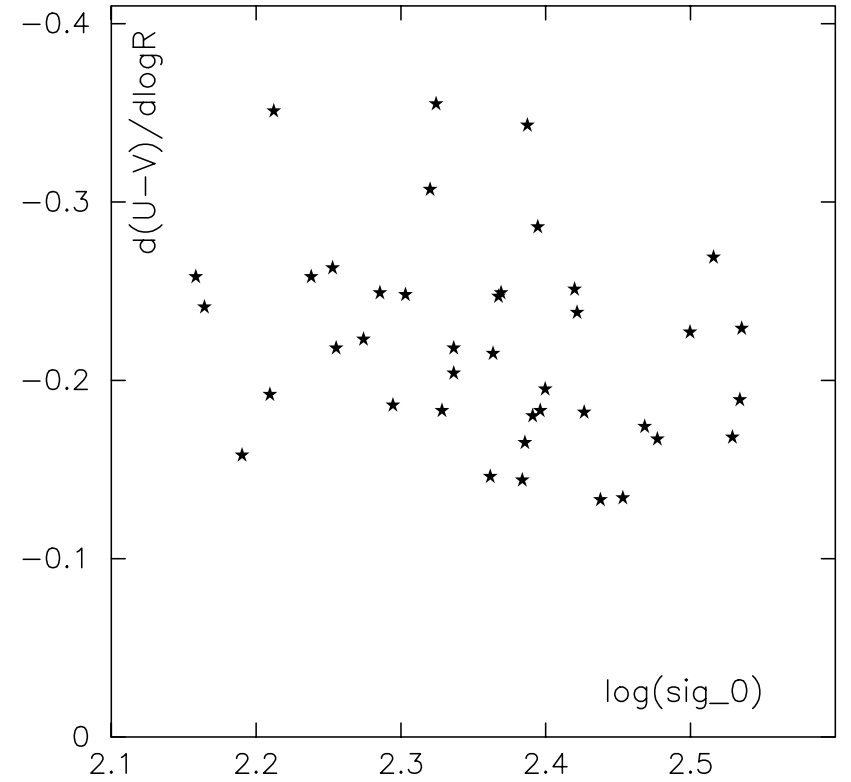

Fig. 4. Correlation diagram between the $\Delta_{U V}$ gradient and the central velocity dispersion $\log \sigma_{0}$. There is an uncertain trend for this gradient to decrease for objects with $\log \sigma_{0}>2.4$. Other colours lead to similar diagrams.

\subsubsection{Gradients for morphologically peculiar E galaxies}

Michard \& Prugniel (2004) studied morphologically "peculiar" E-type galaxies and found 37 so-called Pec objects in a total sample of 114. The Pec subsample had to be subdivided into the $Y P$ family (type NGC 2865), with evidence for the mixing of a younger stellar population with the "normal" old one, plus the NP family (type NGC 3923) devoid of such evidence. In the present study of colour gradients, there are 16 previously classified galaxies of the Pec subsample. The averages for the objects with morphological peculiarities are compared to the average gradients for the complete sample in Table 7, the $Y P$ and $N P$ objects being considered separately. It appears that objects of both families have, in all colours, smaller gradients than the general average. This difference is more marked for the $Y P$ family, at least in $\Delta_{V J}$ and $\Delta_{V K}$. The residuals under discussion are rather marginal from a statistical point of view. If, however, NGC 4125, a dusty object with exceptionnally large $\Delta_{V J}$ and $\Delta_{V K}$ is discarded from the sample of $9 Y P$, the mean of the remaining values becomes much smaller than average and quite significant, i.e. $0.079 \pm .017$ and $0.143 \pm .014$ respectively.

The younger stars in peculiar Es, whose presence is proven from line-and colour indices, are likely to be concentrated near the galaxy center, thus making the central regions bluer and lowering the gradients.

\subsubsection{Gradients for various $E$ subtypes}

According to the results collected in Table 8, the mean values and dispersions of colour gradients are systematically larger for the disky ellipticals than for the boxy objects. The percentage excess in $d i \mathrm{E}$ colour gradients above those pertaining to the $b o \mathrm{E}$ is minimal for $U-B$ and tend to increase with colours 

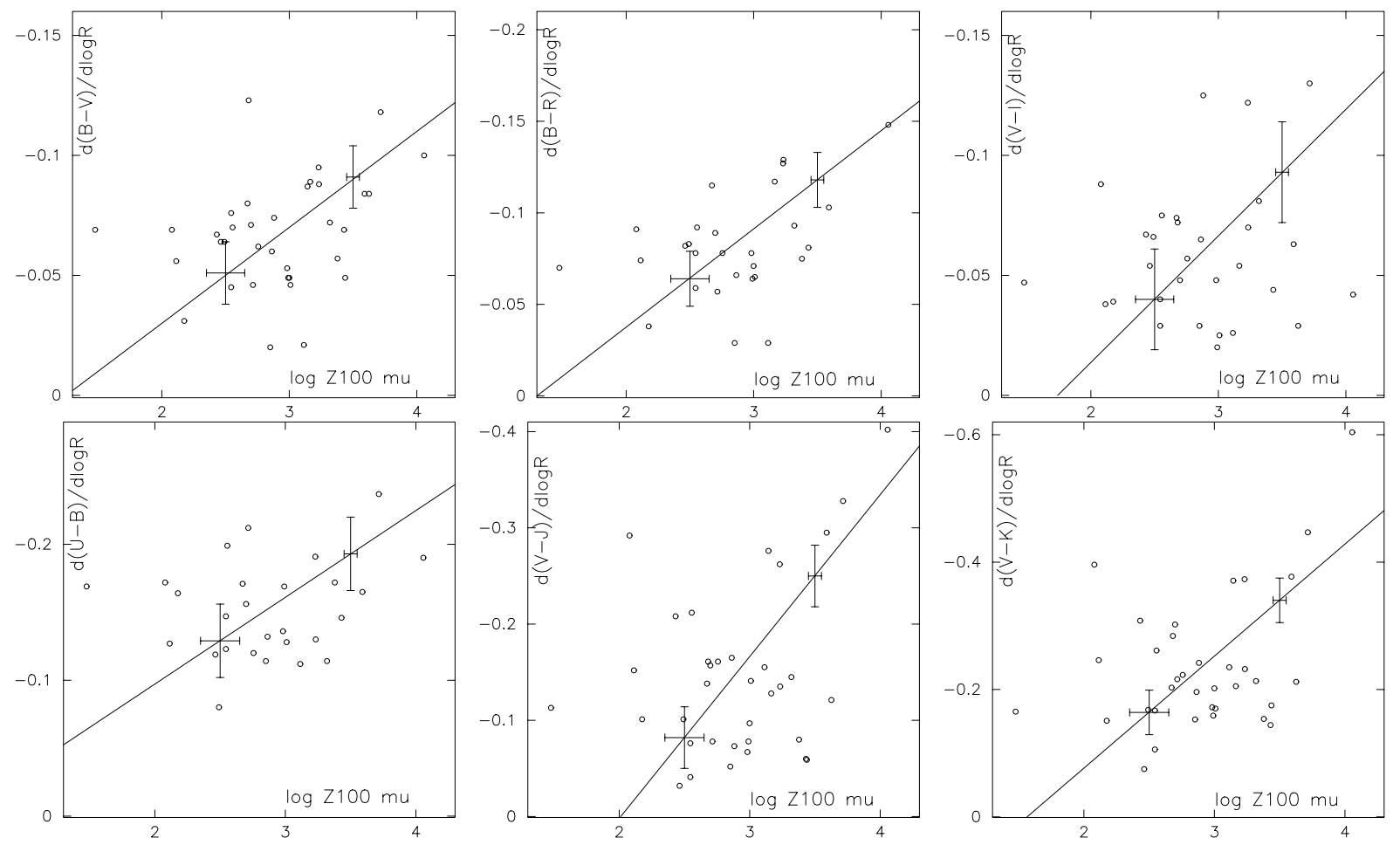

Fig. 5. Correlation diagrams between the IRAS $100 \mu$ flux and gradients in various colours. The fluxes are reduced to the Virgo distance and plotted in a logarithmic scale, after applying a bias of 10 units of the Knapp et al. (1989) scale. Average errors are plotted for two ranges of IRAS flux: they were estimated from a number of individual $\sigma$ values in the quoted catalogue.

Table 7. Comparison of average values of colour gradients for our full sample and for the Pec subsample of morphologically peculiar objects (Michard \& Prugniel 2004). Among Pec objects we distinguish the $Y P$ family with evidence for the presence of some young stars and the $N P$ family with no such evidence.

\begin{tabular}{llllllllll}
\hline \hline $\mathrm{Y}$ & $A l l$ & $N$ & $A \mathrm{v}$ & $Y P$ & $N$ & $A \mathrm{v}$ & $N P$ & $N$ & $A \mathrm{v}$ \\
\hline$\Delta_{B V}$ & $A l l$ & 53 & $-0.066 \pm .003$ & $Y P$ & 9 & $-0.060 \pm .005$ & $N P$ & 7 & $-0.057 \pm .006$ \\
$\Delta_{U B}$ & id & 41 & $-0.157 \pm .007$ & id & 7 & $-0.137 \pm .006$ & id & 6 & $-0.138 \pm .010$ \\
$\Delta_{B R}$ & id & 43 & $-0.082 \pm .004$ & id & 8 & $-0.074 \pm .005$ & id & 6 & $-0.074 \pm .011$ \\
$\Delta_{V I}$ & id & 47 & $-0.061 \pm .005$ & id & 7 & $-0.052 \pm .006$ & id & 7 & $-0.053 \pm .008$ \\
$\Delta_{V J}$ & id & 53 & $-0.136 \pm .013$ & id & 9 & $-0.108 \pm .029$ & id & 7 & $-0.123 \pm .021$ \\
$\Delta_{V K}$ & id & 53 & $-0.227 \pm .015$ & id & 9 & $-0.169 \pm .029$ & id & 7 & $-0.201 \pm .024$ \\
$\Delta_{J K}$ & id & 54 & $-0.093 \pm .004$ & id & 9 & $-0.073 \pm .009$ & id & 7 & $-0.083 \pm .009$ \\
\hline
\end{tabular}

(1) Gradient $Y$; (2) sample; (3) number of objects $N$; (4) average with estimated error; (5) sample; (6) number of objects $N$; (7) average with estimated error; (8) sample; (9) number of objects $N$; (10) average with estimated error.

more sensitive to dust. The larger $d i \mathrm{E}$ gradients are probably due to the influence of dust. Indeed, apparent dust patterns are much more frequent and important in $d i \mathrm{E}$ than $b o \mathrm{E}$ (Michard 1998), the $u n \mathrm{E}$ being intermediate.

\subsection{Possible effects of dust}

- A number of galaxies have remarkably large $\Delta_{V J}$ and $\Delta_{V K}$ gradients. Using as limit the sum (average+sigma) of the above distributions, that is -0.228 in $\Delta_{V J}$ and -0.339 in $\Delta_{V K}$, we find that 7 galaxies have larger gradients in both colours and 10 in $V-K$ only. These objects are of two kinds: i) galaxies with a good amount of dust: NGC 5866, an S0 described in The Hubble Atlas of Galaxies (Sandage 1961) and the diE NGC 2974, 4125, 4278, 4589 (Michard 1998). Other dusty galaxies such as NGC 1052 and 2768 (also $d i \mathrm{E}$ ) have near average gradients; ii) miscellaneous objects without obvious common properties: NGC 720, 3377, 3608, 3962 and 4660. This later object has the largest values of the gradients but for NGC 5866, and since its $\Delta_{V I}$ is also large, it might contain "hidden" dust (unseen by IRAS however).

- We have searched correlations between colour gradients and the estimates of total dust given by IRAS far-IR fluxes. We use here the $100 \mu$ flux from Knapp et al. (1989) corrected to the Virgo distance and plotted in a logarithmic scale. An ad hoc subsample has been formed, retaining only 
Table 8. Mean colour gradients for the subtypes of ellipticals, respectively $d i \mathrm{E}$ and $b o \mathrm{E}$. The $u n \mathrm{E}$ are always intermediate. The S0 NGC 5866 was rejected.

\begin{tabular}{lllll}
\hline \hline Subtype & Grad. & $N$ & Mean & Sigma \\
\hline$d i \mathrm{E}$ & $\Delta_{U B}$ & 12 & $-0.167 \pm .012$ & .042 \\
$b o \mathrm{E}$ & $\Delta_{U B}$ & 13 & $-0.144 \pm .007$ & .025 \\
$d i \mathrm{E}$ & $\Delta_{B V}$ & 20 & $-0.072 \pm .004$ & .020 \\
$b o \mathrm{E}$ & $\Delta_{B V}$ & 14 & $-0.057 \pm .004$ & .015 \\
$d i \mathrm{E}$ & $\Delta_{B R}$ & 12 & $-0.089 \pm .008$ & .028 \\
$b o \mathrm{E}$ & $\Delta_{B R}$ & 13 & $-0.072 \pm .005$ & .019 \\
$d i \mathrm{E}$ & $\Delta_{V I}$ & 17 & $-0.081 \pm .010$ & .041 \\
$b o \mathrm{E}$ & $\Delta_{V I}$ & 13 & $-0.038 \pm .005$ & .019 \\
$d i \mathrm{E}$ & $\Delta_{V J}$ & 19 & $-0.166 \pm .023$ & .100 \\
$b o \mathrm{E}$ & $\Delta_{V J}$ & 14 & $-0.105 \pm .017$ & .064 \\
$d i \mathrm{E}$ & $\Delta_{V K}$ & 19 & $-0.262 \pm .028$ & .121 \\
$b o \mathrm{E}$ & $\Delta_{V K}$ & 14 & $-0.187 \pm .022$ & .081 \\
$d i \mathrm{E}$ & $\Delta_{J K}$ & 20 & $-0.104 \pm .007$ & .031 \\
$b o \mathrm{E}$ & $\Delta_{J K}$ & 14 & $-0.076 \pm .007$ & .028 \\
\hline
\end{tabular}

(1) Subtype; (2) gradient; (3) number of cases; (4) mean; (5) standard deviation.

Table 9. Comparison of average values of colour gradients for the subsample $I R_{0}$ of objects with zero IRAS $100 \mu$ flux and the subsample $I R_{1}$ of objects with non zero flux (Knapp et al. 1989).

\begin{tabular}{lllclll}
\hline \hline$Y$ & $I R_{0}$ & $N$ & $A v_{0}$ & $I R_{1}$ & $N$ & \\
\hline$\Delta_{B V}$ & $I R_{0}$ & 17 & $-0.065 \pm .005$ & $I R_{1}$ & 35 & $-0.067 \pm .004$ \\
$\Delta_{U B}$ & id & 13 & $-0.166 \pm .015$ & id & 27 & $-0.152 \pm .007$ \\
$\Delta_{B R}$ & id & 14 & $-0.083 \pm .008$ & id & 28 & $-0.081 \pm .005$ \\
$\Delta_{V I}$ & id & 15 & $-0.057 \pm .009$ & id & 34 & $-0.063 \pm .006$ \\
$\Delta_{V J}$ & id & 17 & $-0.106 \pm .026$ & id & 35 & $-0.147 \pm .015$ \\
id & id & 16 & $-0.089 \pm .014$ & id & 34 & $-0.139 \pm .013$ \\
$\Delta_{V K}$ & id & 17 & $-0.199 \pm .029$ & id & 35 & $-0.237 \pm .018$ \\
id & id & 16 & $-0.175 \pm .017$ & id & 34 & $-0.227 \pm .015$ \\
$\Delta_{J K}$ & id & 17 & $-0.090 \pm .009$ & id & 35 & $-0.092 \pm .005$ \\
\hline
\end{tabular}

1) Gradient $Y$; (2) subsample $I R_{0}$; (3) $N$ number of objects; (4) average $A v_{0}$; (5) subsample $I R_{1}$; (6) $N$ number of objects; (7) average $A v_{1}$. NB: the 2 nd. line for $\Delta_{V J}, \Delta_{V K}$ gives the solution after the rejection at $3 \sigma$ of NGC 4660 from the subsample $I R_{0}$ and 5866 from $I R_{1}$.

the objects with non-zero IRAS $100 \mu$ flux. Correlation diagrams are shown in Fig. 5, for all the measured colour gradients, but $\Delta_{J H}$ and $\Delta_{J K}$. A modest correlation is indeed apparent in all cases: it nearly vanishes for $\Delta_{V I}$, where the measurements are particularly uncertain. Linear fits to these correlation diagrams have been calculated. The "impartial" solutions are plotted on the figure. The Pearson's coefficient varies between a very low value of 0.12 for $\Delta_{V I}$ to 0.39 for $\Delta_{B V}$ and 0.42 for $\Delta_{B R}$, the gradients with the smallest errors. In Table 9 are compared the averages of the gradients for two subsamples: the objects with zero $100 \mu$ flux reported in the IRAS catalogue on the one hand, and those with a measured flux on the other, i.e. the subsample used in Fig. 5. These two average gradients are nearly equal for $\Delta_{U B}, \Delta_{B V}, \Delta_{B R}, \Delta_{V I}$ and $\Delta_{J K}$. On the other hand, the gradients are slightly larger in the IRAS detected sample for $\Delta_{V J}$ and $\Delta_{V K}$. The gradients in a specific colour belong to the same distribution, or nearly so, wether or not the object contains enough dust to be detected by IRAS. This applies to a sample of generally small IRAS flux and dust content, and does not preclude a few objects to be noticeable for their relatively large gradients $\Delta_{V J}$ and $\Delta_{V K}$ as noted above. These few objects are instrumental in producing the faint correlations detected between the $100 \mu$ flux, when measurable, and the colour gradients.

- If significant amounts of diffuse dust are introduced in otherwise unchanged galaxies, the gradients may be modified in such a way as to contradict some of the observed correlations of Fig. 3. This is the case for the $\Delta_{B R}$ against $\Delta_{B V}$ correlation, which essentially express the fact that $V-R$ is little sensitive to metallicity variations and has very small gradients. From the models of Wal92, the dust induced gradient $\Delta_{V R}$ is -0.018 for a modest dust content $(\operatorname{Tau}(V)=1$ in the authors notation) and would reach -0.05 for a very dusty model $(\operatorname{Tau}(V)=4)$. The presence of a large proportion of objects with such dust amounts would completely destroy the excellent correlation of $\Delta_{B R}$ against $\Delta_{B V}$ established by the IMP02 data.

Summing up the indications from IRAS fluxes and "optical" dust, it appears that dust plays some role in Optical-Near IR colour gradients, but surely not a major one, except perhaps in a minority of dusty galaxies.

\section{Simulated datasets of colour gradients}

The purpose of this exercize is to get insight into the origin of the rather poor correlations between the various colour gradients collected here: these are displayed in Fig. 3 and numerically described in Table 5. Errors of measurement play a major role in jeopardizing these correlations, and are the only source of scatter if the colours are defined by the run of metallicities. "Cosmic" scatter is also introduced if both mean age and metallicity contribute independently (not if the so-called "degeneracy" is complete), or if dust is also significant.

We have calculated the colours profiles of "dummy galaxies", several being consistent with the monolithic scenario, and about 50 for the hierachical scenario. Then gradients have been "measured", giving plausible datasets for dustless objects in either one of the two formation scenarios. The gradients have been modified by the inclusion of some dust according to various object to object distributions. The final datasets were finally polluted by noise as evaluated above (see Table 4).

\subsection{Simulating colour gradients}

\subsubsection{Simulation of populations}

Composite stellar populations with appropriate colour gradients are simulated by superposing 6-12 SSP with various metallicities, ages, radial extent and relative masses $q_{M}$. The tables in W94 and BC03 are used as sources of SSP colours, with 6 metallicity $Z$ and some 20 age values: these are readily recovered from the $\mathrm{BC} 03$ tables, but interpolation between the few available tables is needed when using W94. The projected radial distribution of each SSP is assumed to follow the $r^{1 / 4}$ law, and the de Vaucouleurs radius $r_{\mathrm{e}}$ measures its radial extent.

- It is easy to simulate the results of the monolithic scenario. One may assemble nearly coeval SSP with the $6 Z$ values in 
the $\mathrm{BC} 03$ tables for instance, $Z=0.0001$ being the lesser and $Z=0.05$ the larger, and change the associated values $r_{\mathrm{e}}$ and $q_{M}$. To produce a gradient of the proper sign, $r_{\mathrm{e}}$ is maximal at $Z=0.0001$ and decreases with increasing $Z$. Increasing the range of $r_{\mathrm{e}}$ or the range of $q_{M}$ does increase the colour gradients. Lowering $q_{M}$ at small $Z$ while increasing $q_{M}$ at high $Z$ reddens the colours, so that the predicted relation between gradient and average colour (similar to Chiosi \& Carraro 2002) is readily obtained.

- To mimick the results of the hierarchical scenario the "rules of the game" are more complex. First a "primeval" galaxy is built from 6 nearly coeval SSP, with star formation occuring in the range 13.5-11.0 Gyr ago. This component contains 40 to $70 \%$ of the total mass and may present various gradients left over until the present day: initial population gradients may be destroyed in mergers (Meza et al. 2003) or partly survive these (Wise 1980; Quinn et al. 1990). A constant $r_{\mathrm{e}}$ leaves no gradient in the "primeval" object (or "primary"). As above, to produce a gradient, different $r_{\mathrm{e}}$ values are selected for each population component, those with greater metallicities having lesser radial extents. Contrary to the requirements for the monolithic case however, the primeval population should offer a large variety of gradients with no correlation with average colours. The $r_{\mathrm{e}}$ values used in these simulations are generally 4.0 (arbitrary units) for the very low $Z=0.0001$ component. For the highest $Z=0.050$ population one may keep the same $r_{\mathrm{e}}$, giving an object with zero gradient, or decrease it, down to $r_{\mathrm{e}}=0.3$ for an object with large colour gradients. Obviously the actual gradients depend both on the $r_{\mathrm{e}}$ values and the relative masses $q_{M}$ of the components.

To the primeval object, a series of 1 to 3 "events" are added, corresponding to mergers and starbusts: each event is described with 1 to 3 representative SSP. The populations of merged objects is systematically younger than the stars of the primary and are devoid of very low metallicity stars. The most recent starbust occurs in the 3-10 Gyr age range (Kauffmann et al. 2000) with solar and super-solar metallicity, and its stars remain strongly concentrated $\left(r_{\mathrm{e}}\right.$ between 0.4 and 0.1 ), with a strong influence on the resulting colour gradients. A younger starbust would leave a distinct blue patch, with a large deviation from the colour profiles usually measured.

Varying the mass and gradients of the "primary" population, the number and characters of the subsequent "events", one may obtain a large variety of "dummies", aimed to mimick the build-up of populations, especially their gradients, in the hierarchical scenario. In our opinion, one should not introduce quantitative laws (presently unknown) in some systematic survey of the parameter space: it is sufficient to explore it through a random walk guided by the above noted qualitative rules.

\subsubsection{Dust induced colour gradients}

Wal92 give the centrally emergent light in the usual colours for models of $r^{1 / 4}$ ellipticals containing various amounts of diffuse dust, as measured by the central $V$ optical depth $\tau_{V}$ in the range
$0-4$ (noted $\operatorname{Tau}(V)$ in the quoted paper). The central colour is readily obtained, while the colour at large radii is zero, hence an estimate of the dust induced colour gradients. We have considered a number of possible distribution of $\tau_{V}$ for the above calculated datasets of mock galaxies: constant $\tau_{V}$, triangular peak, maximum at small $\tau_{V}$ with a "tail" of dusty objects. As expected the mean $\tau_{V}$ directly affects the average gradients in the datasets, while the eventual "tail" at larger $\tau_{V}$ would help to explain the relatively few galaxies of remarkably large gradients (see Sects. 3.1 and 3.3). For reasons given in 3.3 our favored model is made up of a subsample of $20 \%$ dusty objects and a main sample of nearly dustless ones, without detectable effects on colour gradients.

\subsection{Results}

Since the datasets of "dummy galaxies" have no other properties than photometric gradients, and were not formed according to very stringent rules, the statistics of the absolute values of their colour gradients are not very robust. The only significant comparison with observations are the "inside correlations" between gradients in different colours (Fig. 3, Table 5). In Fig. 6 are shown, as examples, correlation diagrams of gradients built from the BC03 SSP for the two discussed scenarii: i) without noise nor dust; ii) with noise iii) with a dusty subsample. The present simulations lead to the following remarks:

- The slopes of the correlations between gradients are similar for the large sets of dummy galaxies built in analogy with the hierarchical scenario, and for the group corresponding to the monolithic mechanism (Fig. 6). We believe these slopes to be properties of the SSP theories.

- In the hierarchical option, the correlation diagrams show little dispersion, although both the ages and metallicities of the SSP components are involved, probably due to the classical age-metallicity degeneracy. The dispersion increases however, and curvature in the diagrams occurs at low gradients: these low values are due to the introduction of relatively recent starbusts in the population (Fig. 6).

- The average gradients in various colours are different for datasets built from the two used sources of SSP, i.e. W94 and $\mathrm{BC} 03$, and may actually disagree stongly with the observed ratios. This is also the case of the slopes of the correlation diagrams or equivalently the ratios of different gradients. For instance the average $\left\langle\Delta_{U B}\right\rangle$ is 2.36 times $\left\langle\Delta_{B V}\right\rangle$ in the observed sample, but this same ratio amounts to 2.76 in dustless datasets built from W94 colours and only 1.94 with the BC03 SSP. The introduction of dust will lower the discussed ratio: the disagreement between the observed and calculated ratio values will be increased in simulations from the $\mathrm{BC} 03$ tables, but eventually nullified with the W94 tables and a modest amount of dust.

- Similarly the averages of gradients in the near IR, again relative to $\Delta_{B V}$, increases with the introduction of dust, but these are already larger in the calculated datasets, with both sources of theoretical colours, than in the observed sample. The disagreement between observed and calculated relative average gradients becomes rather extreme for $\Delta_{V J}$, observed much lower than predicted. It appears that the 


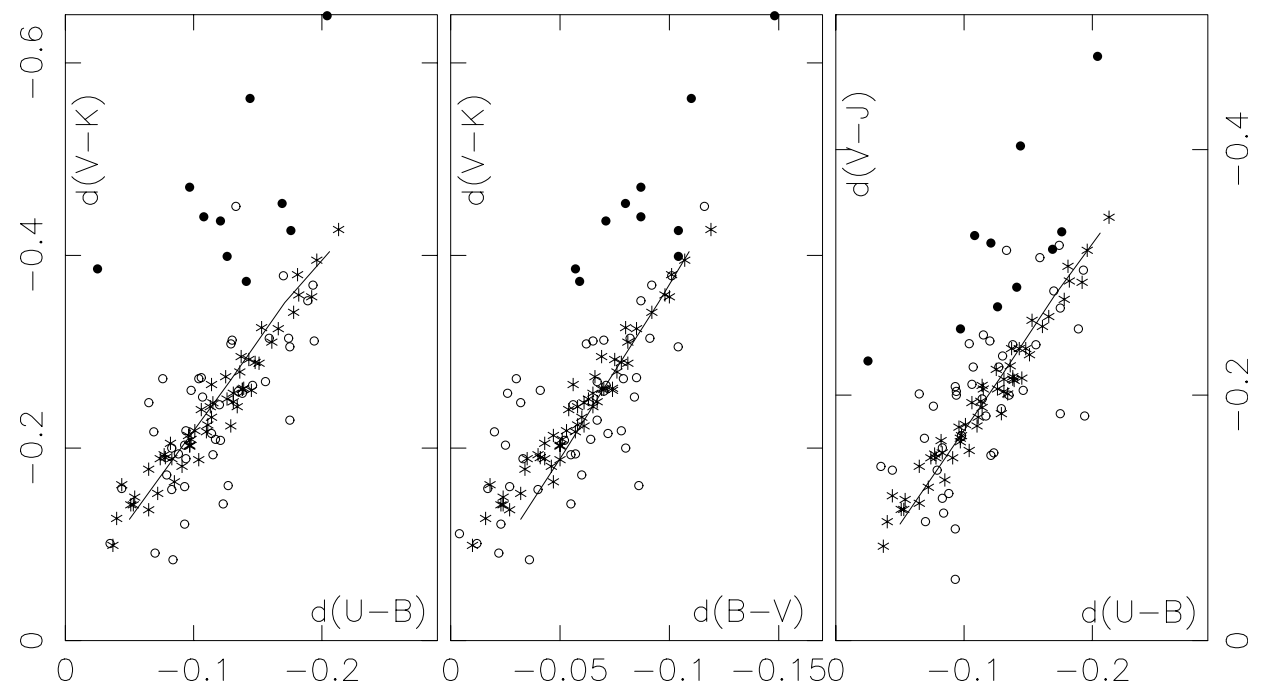

Fig. 6. Correlation diagrams for the pairs of gradients $\Delta_{V K}$ against $\Delta_{U B}$ or against $\Delta_{B V}$, and $\Delta_{V J}$ against $\Delta_{U B}$. Stars: dataset of 53 dummy objects with gradients mimicking the results of the hierarchical scenario. Open circles: subsample of 43 dustless objects affected by noise. Filled circles: subsample of 10 objects with gradients affected by diffuse dust with $\tau_{V}=2$ in models by Wal92 (Tau $(V)$ in their notation) and noise. Line: objects mimicking the monolithic scenario. The dummies are built from SSP in BC03. These correlation diagrams show a significant segregation, often larger than noise, between dustless and dusty objects.

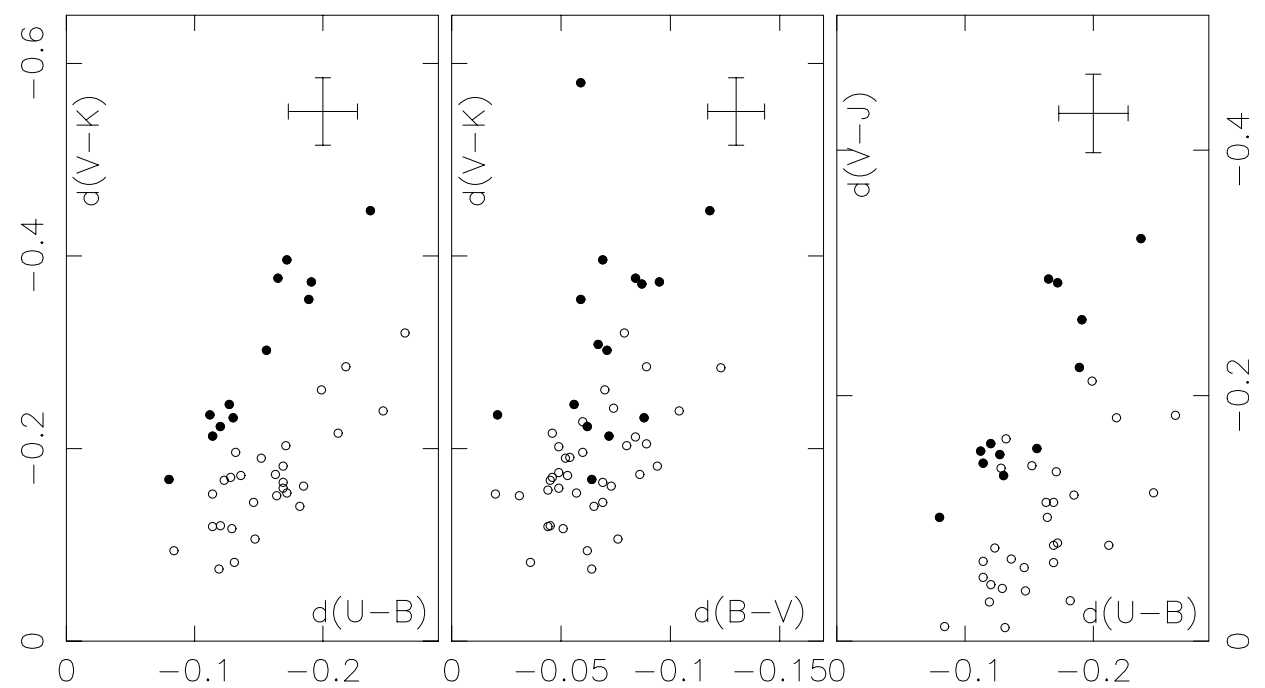

Fig. 7. Correlation diagrams for the pairs of gradients $\Delta_{V K}$ against $\Delta_{U B}$ or against $\Delta_{B V}$, and $\Delta_{V J}$ against $\Delta_{U B}$. Open circles: observed galaxies with no evidence for a significant amount of dust. Filled circles: subsample of objects with gradients possibly affected by diffuse dust. Average observational errors (Table 4) are indicated. The correlation diagram $\Delta_{V K}$ against $\Delta_{U B}$ gives evidence of the expected bimodality between dusty and dustless objects, an evidence which is supported by the other two diagrams. Our estimates of dust content, in terms of models in Wal92, are collected in Table 10. Average obsevational errors are from Table 4.

average dust content in the observed sample should be close to zero, in the sense that adding dust would increase the discrepancies between observed and simulated relative gradients. This conclusion is dependent upon the ability of the used SSP models to represent reasonably well the colours of the real populations at the given age and metallicities.

\subsection{Dust detection from gradients?}

\subsubsection{Experiment with a simulated dataset}

Looking at the correlations diagrams of Fig. 6, we note that the dust affected subsample of 10 objects (filled symbols) is completely separated from the main sample of dustless dummies in the $\Delta_{V K}$ against $\Delta_{U B}$ diagram (with one of these intruding in the same region however): the $\Delta_{V K}$ is the most sensitive to dust of all colour gradients, the $\Delta_{U B}$ the least. This segragation is also present in the other three diagrams, i.e. $\Delta_{V J}-\Delta_{U B}$, $\Delta_{V K}-\Delta_{B V}$ and $\Delta_{V J}-\Delta_{B V}$ (not shown), but the gradient increments due to dust decrease, and may become of the same order as errors of measuremnts. With the assumed $\tau_{V}=2$ these increments are -0.019 in $\Delta_{U B},-0.033$ in $\Delta_{B V},-0.135$ in $\Delta_{V J},-0.206$ in $\Delta_{V K}$. Such diagrams provide a way to recognize the objects, dummies or galaxies, which contain a relatively large amount of dust, from the objects where the ISM has negligible colour effects. Dust induced colour increments could also in principle 
be measured, and interpreted in terms of dust content with the help of the Wal92 models.

An ad hoc algorithm has been used to recover the dusty dummies of Fig. 6 diagrams, standing "well above" the ridge line in the 4 correlation diagrams. By "well above" is meant $1.6 \sigma$ of the correlation after several cycles of rejection of deviating objects. The results are as follows: i) $\Delta_{V K}-\Delta_{U B}$ pattern: all 10 objects recovered, plus 1 intruder; ii) $\Delta_{V J}-\Delta_{U B}: 10$ objects recovered plus 7 intruders; iii) $\Delta_{V K}-\Delta_{B V}: 8$ objects recovered plus 2 intruders; iv) $\Delta_{V J}-\Delta_{B V}$ : not used. Spurious objects retained from one diagram are rejected from the two others, allowing a precious control. From the distance of the data point representing a dusty object to the ridge line of the correlation we estimate its excess gradient: for the $\Delta_{V K}-\Delta_{U B}$ diagram and its 10 dusty objects, the average of the excess gradients is -0.204 (against the exact value of -0.181 ), with a standard deviation of 0.078 , coresponding to a dust content $1<\tau_{V}<3$ according to Wal92, instead of the adopted $\tau_{V}=2$.

\subsubsection{Estimates of the diffuse dust content of observed objects}

Using the same technique, we have tried to find the diffuse dust content, gauged by the $\tau_{V}$ parameter of the Wal92 models, of observed galaxies. This procedure is hampered in our sample of real galaxies by the following limitations: i) some $25 \%$ of the objects lack the most useful $U-B$ data; ii) the ridge line of the correlations for dustless objects is ill defined, possibly because the "noise" is not a random distribution of observational erors: data from various sources, and prone to systematic errors, have been pieced together. On the other hand, the distribution of the dust content in real galaxies has no reason to be strictly bimodal as in the above simulated sample.

In Fig. 7 are plotted for the observed galaxies the same 3 correlation diagrams as shown in Fig. 6 for dummies. The $\Delta_{V K}$ against $\Delta_{U B}$ correlation gives evidence of the expected bimodality, with segregation of dusty and dustless objects, an evidence which is mildly supported by the other diagrams, although poorly in the graph involving $\Delta_{B V}$. Presumably dusty objects have been recovered from the correlations by an ad hoc numerical treatment, and/or by inspection of the graphs. The excess gradients above the values for other objects have been evaluated, and the corresponding $\tau_{V}$ values derived from Wal92 (Table 3E). The results are given in Table 10. As expected this table contains most of the objects noted in Sect. 3.3 for their exceptional $\Delta_{V J}$ and $\Delta_{V K}$.

\section{Concluding summary}

We have collected a list of radial gradients in 8 colours for 53 E-galaxies, reduced to 41 however in $U-B$, using new measurements from 2 MASS and analysing anew published photometric data. The overall accuracy may be better than in previous work. The distributions of colour gradients (Fig. 2, Table 4) are significant, although still badly affected by errors. The same is true of correlations between the values of the various gradients (Fig. 3, Table 5).
Table 10. Estimates of the diffuse dust content in the Tau $(V)$ scale defined by models in Witt et al. (1992, Wal92), using the correlations $\Delta_{V K}-\Delta_{U B}, \Delta_{V J}-\Delta_{U B}, \Delta_{V K}-\Delta_{B V}$, and $\Delta_{V J}-\Delta_{B V}$ (see Sect. 4.2). Doubtfull cases are noted with a colon.

\begin{tabular}{lllll}
\hline \hline NGC & $n$ & $\tau_{V}$ & $\sigma$ & Notes \\
\hline 1052 & 3 & 0.7 & 0.5 & - \\
1404 & 3 & $0.6:$ & 0.5 & - \\
1537 & 1 & $2.0:$ & 0.7 & noUB \\
1549 & 3 & 1.3 & 0.3 & - \\
2768 & 3 & 1.0 & 0.6 & - \\
2974 & 3 & 2.1 & 0.4 & - \\
2986 & 3 & $1.8:$ & 0.6 & smBV \\
3377 & 3 & 2.9 & 0.5 & - \\
3608 & 3 & $1.8:$ & 0.8 & - \\
4125 & 3 & 2.9 & 0.7 & - \\
4261 & 3 & 1.2 & 0.3 & - \\
4278 & 3 & 1.8 & 0.4 & - \\
4365 & 3 & 1.2 & 0.7 & - \\
4589 & 1 & $2.3:$ & - & noUB \\
4660 & 1 & $>4 .:$ & - & noUB, smBV \\
\hline
\end{tabular}

(1): NGC; (2) $n$ number of usable correlations. This is generally 3 but may be reduced to 1 in a few cases; (3) $\tau_{V}$ : $\operatorname{Tau}(V)$ parameter; (4) $\sigma$ : standard deviation; (5) notes. Notes: noUB no $U-B$ data; $\operatorname{smBV} \Delta_{B V}$ too small for assumed $\tau_{V}$.

To produce a colour gradient, if the role of dust is indeed marginal, the galaxy should contain star populations of various spectral properties, and these should not be fully mixed. It seems well established that metallicity is the main parameter whose radial variations explain the colour gradients. This is indicated by the relative values of the gradients in different colours (Table 6) and also by studies of line-indices (Kobayashi \& Arimoto 1999; Henry \& Worthey 1999).

We believe however, that age variation is a second parameter needed to explain the extraordinary variety of colour gradients in otherwise similar E-objects. It may be expected that stars of different metallicities were also formed at different epochs, only slightly different in the initial episode of star formation, and much more varied in later (merger associated) starbusts. The "monolithic scheme", where stellar generations occur in a rather short and continuous sequence should lead to systematic properties of colour gradients, probably with the same values in objects of the same mass. Current models (Chiosi \& Carraro 2002) predict metallicity gradients increasing with galaxy mass, a prediction which is inconsistent with observations (Fig. 4).

To introduce the observed scatter into the colour gradients, several uncorrelated bursts of star formation are needed, in such dynamical circumstances that no complete mixing can be attained in the elapsed time. The favoured site of stellar formation is probably near the galaxy center where the ISM tends to concentrate. The persistence of stars in this region may be extremely long in a fast rotating disk, whether the regular disk of a $d i \mathrm{E}$ or a Kinematically Distinct Core. It may be much shorter if the new population is stirred into the largely radial orbits of ellipticals by one or repeated subsequent interactions.

Such age effcts have been found for the subsample of Pec galaxies i.e. those with morphological peculiarities 
(Michard \& Prugniel 2004): they have systematically smaller gradients than the complete sample (Table 7) probably due to the presence of younger stars towards the center. This phenomenon is slightly more prononced for the $Y P$ family, those showing, from line- and colour indices, some direct evidence for the existence of these younger stars.

In defining colour gradients, the dust in E galaxies does not play the important role proposed by some authors, at least for the average elliptical. However the gradients correlate slightly with the measured IRAS $100 \mu$ flux (Fig. 5). On the other hand, the mean gradients are nearly the same for the two subsamples of objects detected or undetected by IRAS (Table 7) except perhaps in $V-J$ and $V-K$, the most "dust sensitive" colours. This apparent contradiction can be resolved when considering that colour gradients are affected by dust in a small minority of $o b$ jects. Most of these can be recognized from their noticeable optical dust patterns (see Sect. 3.2).

Sets of dummy objects mimicking the stellar populations in the hierarchical scenario have been calculated and used to test the feasibility of measuring the diffuse dust content of ellipticals: models in Wal92 are used to relate gradients to dust. The correlations in Fig. 6 appear promising for this purpose, especially the $\Delta_{V K}-\Delta_{U B}$ one, between the gradients most sensitive and least sensitive to dust. A preliminary attempt is presented to apply the technique to observed objects (Fig. 7 and Table 10). With better observations, especially in $U-B$, colour gradients might bring new information on the dust remaining in E-galaxies, in complement to far-IR future observations.

Acknowledgements. This research has made use of the NASA/IPAC Infrared Science Archive, which is operated by the Jet Propulsion Laboratory, California Institute of Technology, under contract with the National Aeronautics and Space Administration.

The kind attention of Dr. Jarrett is gratefully acknowledged. I am thankful to Dr. F Combes for helpful advice.

\section{References}

Barnes, J. R. 1992, ApJ, 393, 484

Barnes, J. R., \& Hernquist, L. 1992, ARA\&A, 30, 705

Barnes, J. R., \& Hernquist, L. 1996, ApJ, 471, 115

Bressan, A., Chiosi, C., \& Fagotto, F. 1994, ApJS, 94, 63

Bressan, A., Chiosi, C., \& Tantalo, R. 1996, A\&A, 311, 425

Bruzual, G., \& Charlot, S. 2003, MNRAS, 344, 1000 (BC03)

Carlberg, R. G. 1984, ApJ, 286, 403

Carlberg, R. G. 1984, ApJ, 286, 416

Carter, D. 1978, MNRAS, 182, 797, A\&A, 784

Chiosi, C., \& Carraro, G. 2002, MNRAS, 335, 335

Franx, M., Illingworth, G., \& Heckman, T. 1989, AJ, 98, 538 (Fal89)

Goudfrooij, P., Hansen, L., Jorgensen, H. E., et al. 1994a, A\&AS, 104, 179 (Gal94)

Goudfrooij, P., Hansen, L., Jorgensen, H. E., et al. 1994b, A\&AS, 105, 341
Goudfrooij, P., Hansen, L., Jorgensen, H. E., et al. 1994c, MNRAS, 271,833

Goudfrooij, P., \& de Jong, T. 1995, A\&A, 298, 784

Hatton, S., Devriendt, J. E. G., Ninin, S., et al. 2003, MNRAS, 343, 75

Helly, J. C., Cole, S., Frenk, C. S., et al. 2003, MNRAS, 338, 903

Henry, R. B. C., \& Worthey, G. 1999, PASP, 111, 919

Idiart, T., Michard, R., \& de Freitas Pacheco, J. 2002, A\&A, 383, 30 (IMP02)

Idiart, T., Michard, R., \& de Freitas Pacheco, J. 2003, A\&A, 398, 949

Jarrett, T. H., Chester, T., Cutri, R., et al. 2003, AS, 125, 525

Kauffmann, G., Colberg, J. M., Diaferio, A., et al. 1999, MNRAS, 303, 188

Kauffmann, G., Charlot, S., \& Haenelt, M. 2000, Phil. Trans. R. Soc. London Ser. A, 358, 2121

Kawata, D. 2001a, ApJ, 548,703

Kawata, D. 2001b, ApJ, 558, 598

Kobayashi, C., \& Arimoto, N. 1999, ApJ, 527, 573

Kobayashi, C. 2004, MNRAS, 347, 740

Knapp, G. R., Guathakurta, P., Kim, D. W., \& Jura, M. 1989, ApJS, 70, 329

Larson, R. B. 1969, MNRAS, 145, 405

Larson, R. B. 1974, MNRAS, 166, 385

Mannucci, F., Basile, F., Poggianti, B. M., et al. 2001, MNRAS, 326, 745

Meza, A., Navarro, J. F., Steinmetz, M., et al. 2003, ApJ, 590, 619

Michard, R. 1998, A\&A, 334, 453

Michard, R. 1999, A\&AS, 137, 245 (RM99)

Michard, R. 2000, A\&A, 360, 85 (RM00)

Michard, R. 2005, A\&A, 429, 819

Michard, R., \& Marchal, J. 1994, A\&AS, 105, 481 (MM94)

Michard, R., \& Prugniel, P. 2004, A\&A, 423, 833

Peletier, R. F., Davies, R. L., Illingworth, G. D., et al. 1990, AJ, 100, 1091 (Pal90)

Poggianti, B. M. 1997, A\&AS, 122, 399

Poulain, P. 1986, A\&AS, 64, 225

Poulain, P. 1988, A\&AS, 72, 215

Poulain, P., \& Nieto, J.-L. 1994, A\&AS, 103, 573

Prugniel, P., \& Simien, F. 1996, A\&A, 309, 749 (PS96)

Prugniel, P., \& Héraudeau, P. 1998, A\&AS, 128, 299

Quinn, P. J., Zurek, W. H., Salmon, J. K., et al. 1990, in Dynamics and Interactions of Galaxies, ed. R. Wielen (Springer Verlag)

Rieke, G. H., \& Lebofsky, M. J. 1985, ApJ, 288, 618

Sandage, A. 1961, The Hubble Atlas of Galaxies, Carnegie Inst. of Washington

Schweizer, F., Seitzer, P., Faber, S. M., et al. 1990, ApJ, 364, L33

Schweizer, F., \& Seitzer, P. 1992, AJ, 104, 1039 (SS92)

Silva, D. R., \& Elston, R. 1994, ApJ, 428, 511

White, S. D. M., \& Rees, M. 1978, MNRAS, 183, 341

White, S. D. M. 1980, 191, 1P

Wise, M. W., \& Silva, D. R. 1996, ApJ, 461, 155

Witt, A., Thronson, H. A. Jr, \& Capuano, J. M. 1992, ApJ, 393, 611 (Wa192)

Worthey, G. 1994, ApJS, 95, 107 (W94) 